\title{
Formation of rhabdophane-structured lanthanum orthophosphate nanoparticles in an impinging-jets microreactor and rheological properties of sols based on them
}

\author{
O. V. Proskurina ${ }^{1,2}$, E. V. Sivtsov ${ }^{1}$, M. O. Enikeeva ${ }^{1,2}$, A. A. Sirotkin ${ }^{1}$, R. Sh. Abiev ${ }^{1}$, V. V. Gusarov ${ }^{2}$ \\ ${ }^{1}$ Saint Petersburg State Institute of Technology, Moskovsky Pr., 26, Saint Petersburg, 190013, Russia \\ ${ }^{2}$ Ioffe Institute, Politekhnicheskaya Saint 26, Saint Petersburg, 194021, Russia \\ proskurinaov@mail.ru,pjeka@yahoo.fr, abiev.rufat@gmail.com, victor.v.gusarov@gmail.com
}

DOI 10.17586/2220-8054-2019-10-2-206-214

\begin{abstract}
A free impinging-jets microreactor was used for synthesizing rhabdophane-structured $\mathrm{LaPO}_{4}$ sols. The rheological behavior was investigated for the sols obtained both by reagents mixing in a microreactor, and by pouring the initial solutions together and mixing them on a magnetic stirrer. Lanthanum phosphate sols obtained by two ways are structured systems characterized by deformation behavior accompanied by shear liquefaction. Some discovered anomalies were found to be associated with flow nonequilibrium at low shear rates, which indirectly indicates stronger binding of particles in the structure of samples obtained by the microreactor synthesis.
\end{abstract}

Keywords: $\mathrm{LaPO}_{4} \cdot n \mathrm{H}_{2} \mathrm{O}$, rhabdophane, nanorods, free impinging-jets microreactor, rheology.

Received: 2 March 2019

Revised: 19 March 2019

\section{Introduction}

When aqueous solutions of lanthanum salts interact with phosphoric acid or with solutions of phosphate salts at relatively low temperatures, usually there occurs crystallization of a lanthanum orthophosphate-based phase with a rhabdophane-type hexagonal structure, which contains some amount of $\mathrm{LaPO}_{4} \cdot n \mathrm{H}_{2} \mathrm{O}$ [1-5]. When precipitating in an acidic medium, the rhabdophane-structured nanoparticles are generally shaped as nanorods $[6,7]$.

The morphology, size and structure of nanoparticles, as well as the presence and amount of impurity phases in a nanopowder can significantly influence the mechanical and functional properties of the materials obtained from them $[8,9]$, including the properties of monazite ceramics obtained by sintering nanopowders based on the rhabdophane-structured phase [10-15]. The morphology and size of rhabdophane-structured lanthanum orthophosphate-based particles greatly determine the rheological properties of their dispersion in liquid media and, consequently, the possibility of using nanoparticles as liquid crystals, which, as was shown in [16-19], can be promising when doping $\mathrm{LaPO}_{4}$ with $\mathrm{Eu}^{3+}, \mathrm{Tb}^{3+}$ and other ions. The literature contains a limited number of works on the rheology of a dispersion based on the rhabdophane-structured lanthanum orthophosphate nanoparticles [20].

The mentioned reasons show the necessity to develop such methods for synthesizing lanthanum orthophosphate nanopowders that would ensure their morphological characteristics, dispersion and phase composition necessary for obtaining materials with high-level properties. There exist several methods for synthesizing lanthanum orthophosphate nanoparticles, for example, the sol-gel method [21-23], hydrothermal synthesis [24, 24,25], and the microwave heating synthesis [26-28].

One of the burgeoning trends in chemical technology is the use of microreactors [29]. Impinging-jets microreactors have not yet been sufficiently studied, but have already proved to be promising in the synthesis of nanoparticles [30-34].

Impinging-jet microreactors allow the dissipation of a large amount of energy within a very small volume [35]. Under certain flow regimes of the colliding jets of reagent solutions, it leads to localization of the reaction zone in a confined space with dimensions on the order of hundreds of nanometers, with a uniform distribution of the initial components corresponding to a specified stoichiometry [36]. Under the conditions like used in [34], a nanoparticle of only one phase can be formed, and it will have a size comparable to that of the critical nucleus. As a result, the formation of by-products that differ compositionally from the stoichiometry of the target phase is almost completely excluded. These reasons indicate the promise of using impinging-jet microreactors for producing nanoparticles.

At the same time, the synthesis of lanthanum orthophosphate nanoparticles in a free impinging-jet microreactor (FIJMR) has a significant difference in contrast to the synthesis of cobalt ferrite and bismuth orthoferrite considered in [34]. During the synthesis of $\mathrm{CoFe}_{2} \mathrm{O}_{4}$ and $\mathrm{BiFeO}_{3}$ nanoparticles, a jet of a solution of salts of the initial components, mixed in a specified proportion, collided in the microreactor with a jet containing a precipitant: 
ammonia or sodium hydroxide solution. In this case, there was no need in a precise observance of the proportion between the content of a mixed salts solution and that of the alkali solution in the reaction zone. In the case of lanthanum orthophosphate synthesis in the free impinging-jets microreactor, even a slight deviation from the La:P = $1: 1$ ratio in the reaction zone can lead to the formation of impurity phases with another La:P ratio [37-39]. It may be much more difficult to ensure the necessary La: P ratio in the case of lanthanum orthophosphate synthesis in an impinging-jet microreactor, since in this case solutions containing a lanthanum salt and a phosphate salt (or phosphoric acid) will be supplied into the reaction zone as different jets, the flow rate of which may differ by a few percent.

The prospects of using the thus-synthesized nanoparticles for obtaining nanopowders or compact materials are largely determined by their morphological characteristics, chemical, phase and dispersion composition, as well as by the rheological properties of sols based on them. Recently, interest has emerged in the development and investigation of the different quasi-one-dimensional structures (nanorods, nanowires, nanofibers, nanotubes) is due to their remarkable functional properties [40-44].

These reasons show the urgency of studying the possibility of synthesizing lanthanum orthophosphate nanoparticles in the FIJMR, and of conducting a comparative analysis of characteristics of nanoparticles obtained in a microreactor and of the rheological behavior of sols based on them, compared to the properties of lanthanum orthophosphate nanoparticles obtained by one of the most common synthesis methods.

\section{Experimental}

\section{1. $\mathrm{LaPO}_{4} \cdot n \mathrm{H}_{2} \mathrm{O}$ synthesis}

The initial reagents used in this work were lanthanum nitrate (puriss. grade) and monosubstituted ammonium phosphate $\mathrm{NH}_{4} \mathrm{H}_{2} \mathrm{PO}_{4}$ (pur. grade). The lanthanum phosphate particles were precipitated when pouring $100 \mathrm{ml}$ of $0.064 \mathrm{M}$ aqueous solution of $\mathrm{La}\left(\mathrm{NO}_{3}\right)_{3}$ into $100 \mathrm{ml}$ of $0.064 \mathrm{M}$ aqueous solution of $\mathrm{NH}_{4} \mathrm{H}_{2} \mathrm{PO}_{4}$ and mixing them with a magnetic stirrer. This resulted in the formation of a white suspension, from which, after stirring for a few minutes, a semi-transparent sol was obtained.

Similar solutions were used for the synthesis of lanthanum phosphate particles in a FIJMR. The FIJMR described in [32] was used. The initial solutions were fed into the jet microreactor through two nozzles with a diameter of $0.44 \mathrm{~mm}$ and $0.46 \mathrm{~mm}$. Lanthanum nitrate and ammonium phosphate solutions were supplied as thin jets with a fixed flow rate of $250 \mathrm{ml} / \mathrm{min}$, colliding at a speed of about $23 \mathrm{~m} / \mathrm{s}$ in a vertical plane at an angle of about $72^{\circ}$, at $22^{\circ} \mathrm{C}$ and atmospheric pressure. The mutual arrangement of the nozzles and the flow rate were set to make the collision of jets produce a liquid sheet with an average thickness of $10-15 \mu \mathrm{m}$, in which the initial component solutions contacted each other and mixed (Fig. 1). Co-precipitation of lanthanum phosphate in a jet microreactor occurred within 5-10 milliseconds.

The result was a suspension, which, at stirring, also transformed into a stable sol.

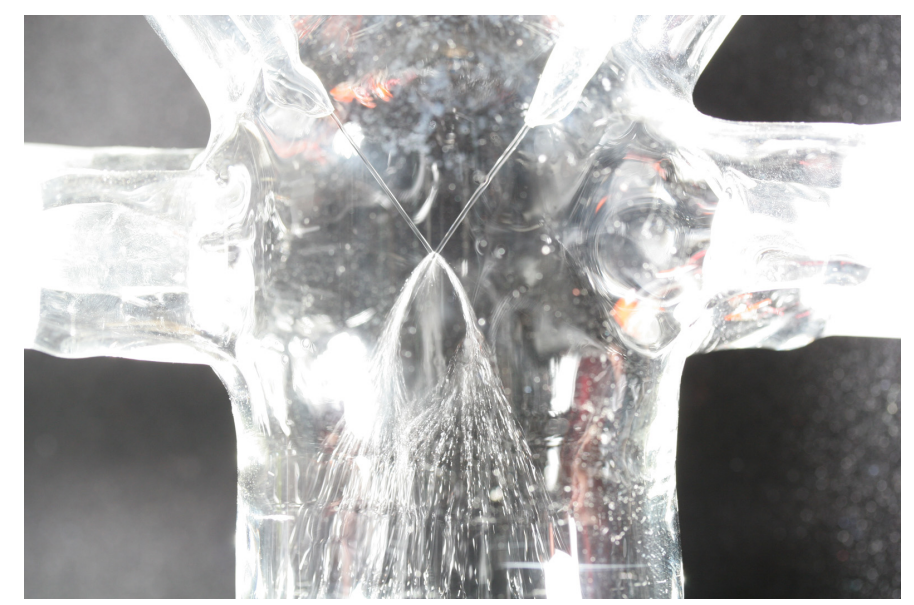

FIG. 1. Liquid sheet formation at the jets collision in the free impinging-jets microreactor

A centrifuge was used to precipitate and wash the obtained samples. The sols were centrifuged for 5 hours at 15 thousand rpm. After 5 cycles of centrifugation, washing with distilled water and using an ultrasonic bath for the dispersal, the obtained samples were dried at $105^{\circ} \mathrm{C}$. 


\section{Characterization}

X-ray diffractograms were recorded on a Rigaku SmartLab 3 powder diffractometer $\left(\mathrm{Cu}_{K \alpha}\right.$ emission) in the $2 \theta=20-60^{\circ}$ angle range with a $0.01^{\circ}$ step and a shooting speed of $0.1^{\circ} / \mathrm{min}$. The samples' phase compositions were found using the ICSD PDF-2 database.

The average crystallite size was determined with the help of the SmartLab Studio II software package from Rigaku. Crystallite size distribution and distribution parameters were determined by the fundamental parameters method in approximation to the log-normal distribution model, using the SmartLab Studio II software package.

The morphology of the washed and dried samples, as well as the particle size and elemental composition of the samples were determined using a Tescan Vega $3 \mathrm{SBH}$ scanning electron microscope with an Oxford Instruments X-ray microanalysis attachment.

Transmission electron microscopy (TEM) studies for determining samples microdiffraction were performed on a JEOL JEM-2100F microscope with the accelerating voltage of $200 \mathrm{kV}$.

The flow curves for the sols were obtained on the Anton Paar PHYSICA MCR302 rheometer, and the data processing was performed using the RHEOPLUS / 32 V.3.62 software. A cone-plane measuring system with a diameter of $25 \mathrm{~mm}$ was used; the minimum gap size was $0.051 \mathrm{~mm}$. The time interval between successive measurements in constructing the flow curve was $2 \mathrm{~s}$.

\section{Results and discussion}

The X-ray diffraction data for the samples are shown in Fig. 2a. Diffractograms of all samples correspond to those of the rhabdophane phase.

The X-ray microanalysis data for the samples show that the P: La ratio in the samples is in the range of (48 \pm 2$):(52 \pm 2)$ at.\%, which, within the limits of error, corresponding to the ratio specified for the synthesis, which corresponds to $\mathrm{LaPO}_{4}$ stoichiometry. A small amount of nitrogen (around 1.8\%) is present in the samples, which may indicate that the ammonium nitrate produced during the synthesis was not completely washed off from the sol particles.
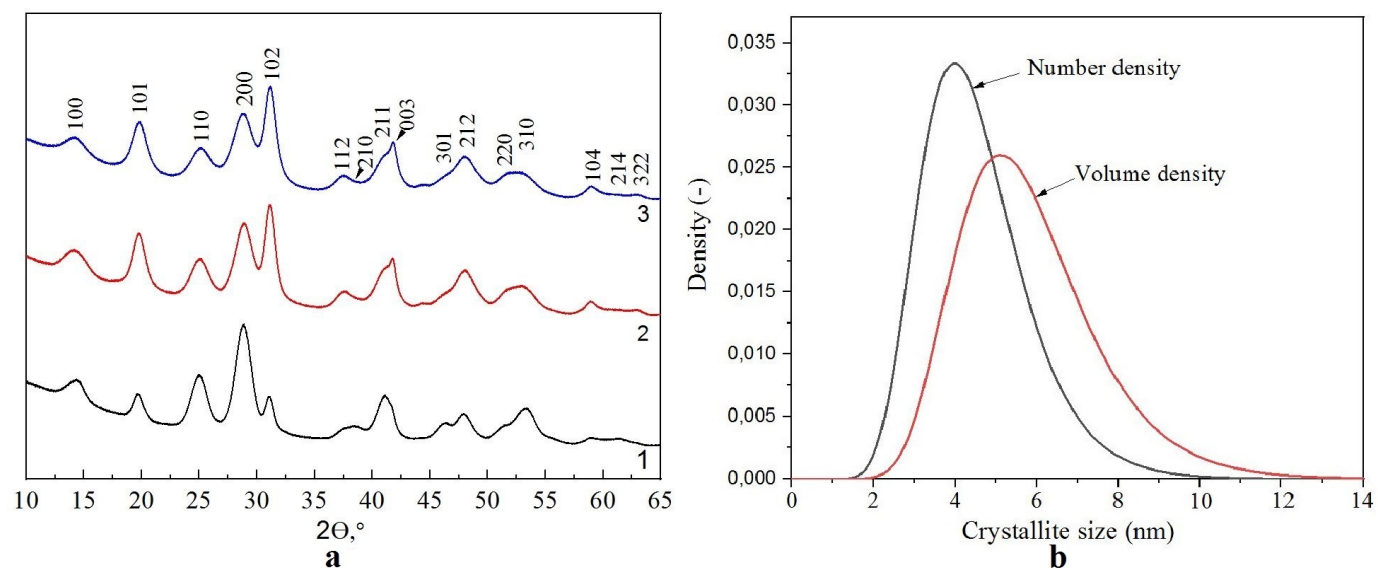

FIG. 2. (a) - X-ray diffraction data: 1 - sol, washed after FIJMR, non-dried, 2 - sol, washed and dried after FIJMR, 3 - sol, washed and dried after precipitation on magnetic stirrer; (b) Distribution of the crystallites number and volume by size, determined from reflex (200) for sample 1

The TEM results presented in Fig. 3 show that individual nanorods are approximately 4-7 nm thick. This result correlates well with the data on the crystallite size distribution obtained from the analysis of the broadened (200) line in the X-ray diffractogram (Fig. 2b). The nanorod length starts from 25-30 nm and reaches several hundred $\mathrm{nm}$. Fig. $3 \mathrm{c}$ demonstrates the oriented coalescence of three hexagonal nanorods along the edges.

Micrographs of samples taken with a scanning electron microscope are shown in Fig. 4. The samples are represented by rods with a thickness of about 20-40 nm and a length of up to $1 \mu \mathrm{m}$.

A comparison of the SEM particle size data with the TEM data and the results of the crystallites size distribution analysis allows a conclusion that $\mathrm{LaPO}_{4} \cdot n \mathrm{H}_{2} \mathrm{O}$ nanocrystals form aggregates consisting of crystallites that are fused together in the form of nanorods oriented along the 6th-order axis of the rhabdophane structure. 


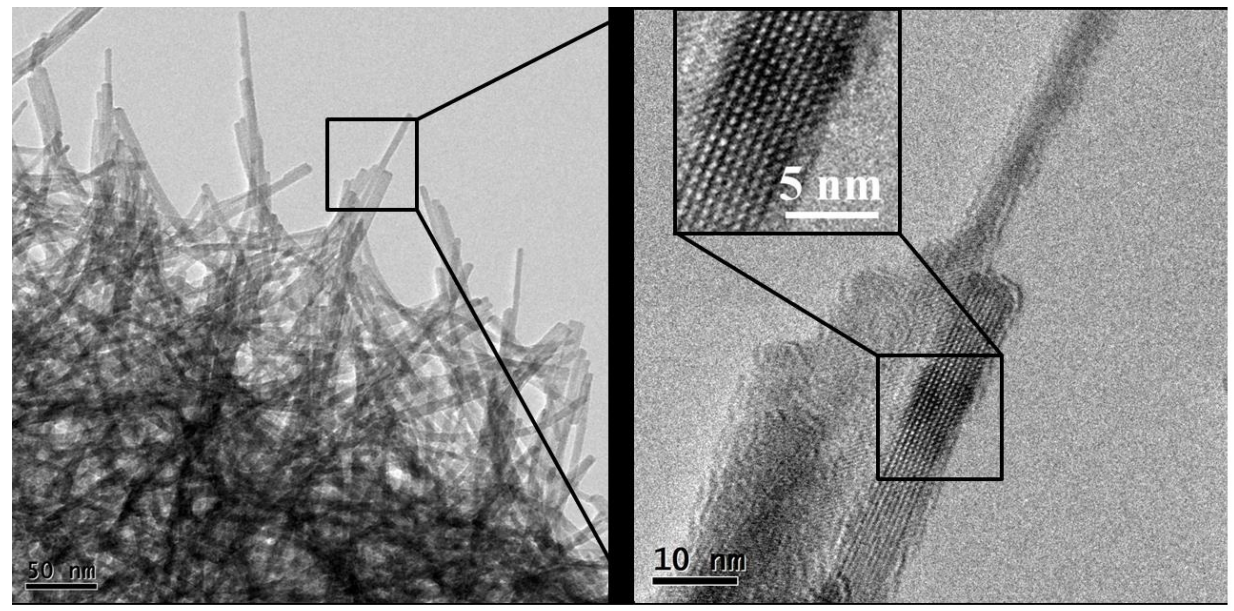

a
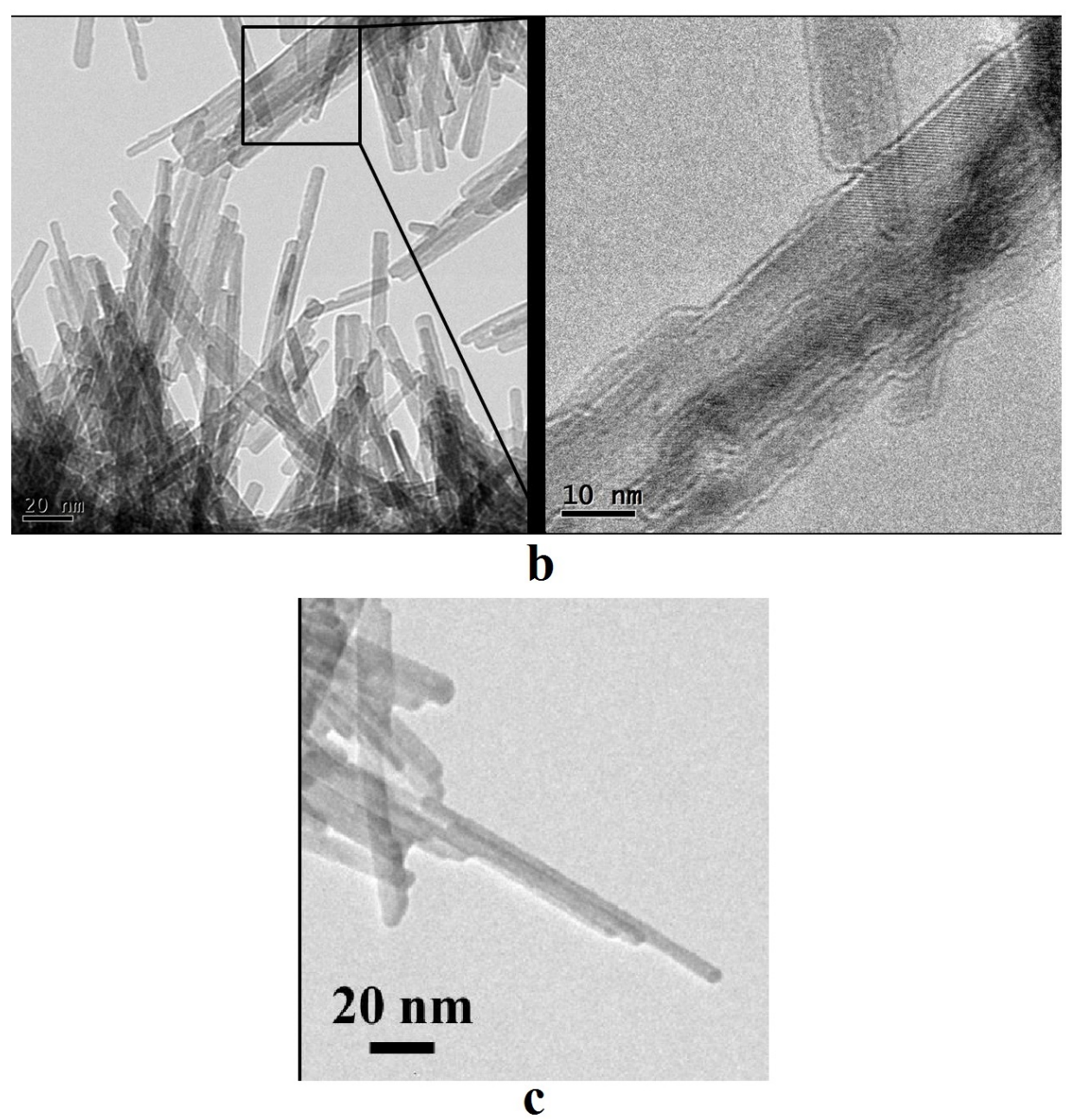

FIG. 3. TEM images for the sample obtained in FIJMR (a), and the sample obtained via precipitation on magnetic stirrer (b), (c) 


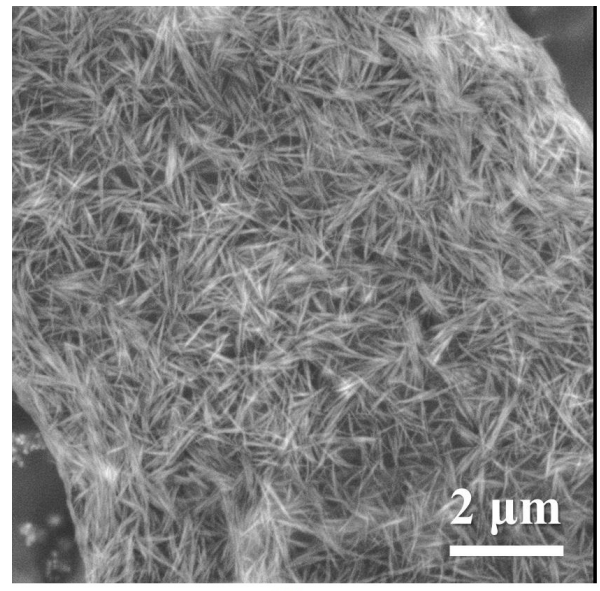

a

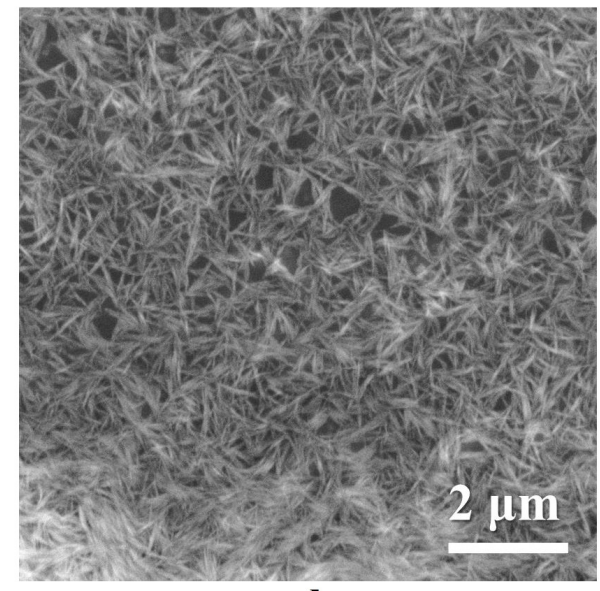

b

FIG. 4. SEM micrographs of the sample obtained in FIJMR (a), and the sample obtained via mixing on magnetic stirrer (b)

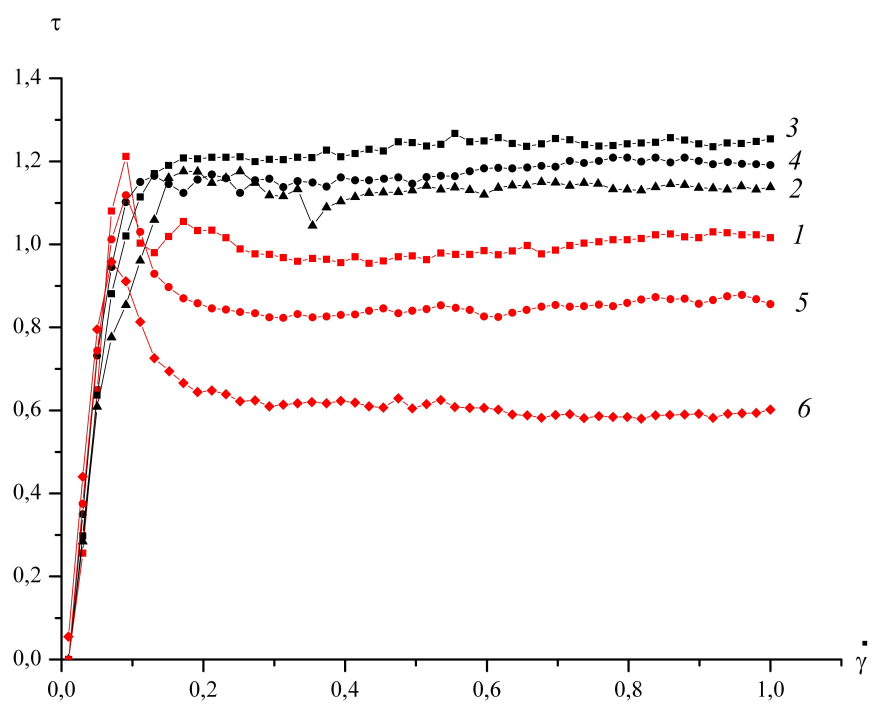

FIG. 5. $\tau(\mathrm{Pa})$ as a function of the shear rate $\gamma\left(\mathrm{s}^{-1}\right)$ for the sols obtained through the microreactor synthesis in the region of low shear rates. Time intervals between adjacent measurements: 1-2, 2-3, 3-4 - 3 min; 4-5 - 6 min, 5-6 - 3 min

The rheological behavior of lanthanum phosphate sols has been studied in two ranges of shear strain rates: at low rates from 0.01 to $1 \mathrm{~s}^{-1}$, and in the range from 0.5 to $200 \mathrm{~s}^{-1}$ which is typical for similar studies. The flow curve was taken at least 4 times for each experiment. The curve numbers match the numbers of the experiments in this series.

The initial series of the flow curves for the sols obtained by microreactor synthesis is characterized by an anomaly, which is expressed in the constant shear stress plateau after a short region of the usual shear-thinning behavior (Fig. 5). Moreover, this change of the flow mode occurs in some cases through the maximum shear stress (Fig. 5, curves 1, 2, 5, 6). This is characteristic of the structured thixotropic systems in a non-equilibrium state during the experiment. As the shear rate increases (when its values are small), the aggregates do not have enough time to collapse to a size that is in equilibrium at a given shear rate, which leads to stress accumulation. At a certain moment, rapid fragmentation of the non-equilibrium aggregates begins and determines the behavior shown in Fig. 1. It is characteristic that a series of successive repetitions of the experiment with the minimum time interval between them leads to the unification of the flow curves (Fig. 5, curves 3,4), which disappears again after a relatively long pause (Fig. 5, curves 5, 6). 


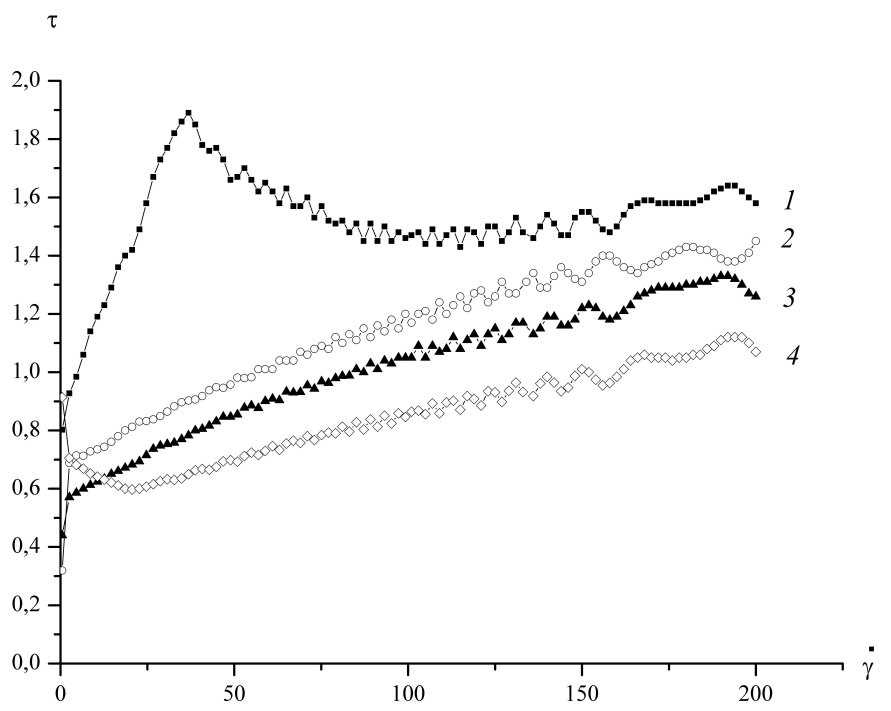

FIG. 6. $\tau(\mathrm{Pa})$ as a function of the shear rate $\gamma\left(\mathrm{s}^{-1}\right)$ for the sols obtained through the microreactor synthesis in a broad range of shear rates. The first curve starts $3 \mathrm{~min}$ after the last measurement in the previous series (Fig. 5). Time intervals between adjacent measurements: 1-2 - 4 min; 2-3 $5 \mathrm{~min} ; 3-4-6 \min$

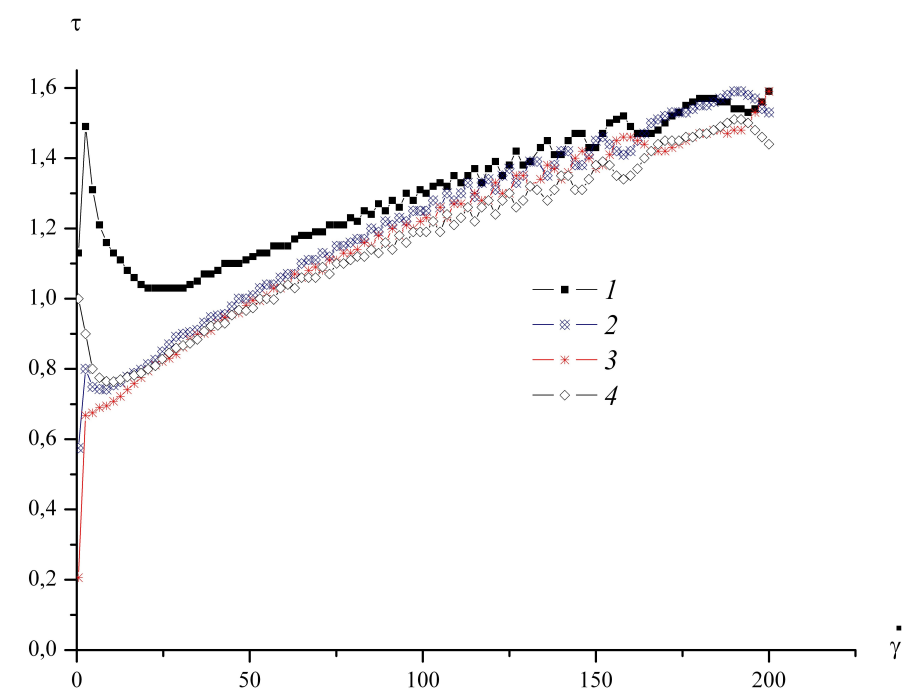

FIG. 7. $\tau(\mathrm{Pa})$ as a function of the shear rate $\gamma\left(\mathrm{s}^{-1}\right)$ for the sols obtained through the microreactor synthesis without deformation prehistory, in a broad range of shear rates. Time intervals between adjacent measurements: 5 min

Interestingly, the replacement of one sample with another that has no deformation prehistory does not lead to a change in the flow curves appearance (Fig. 7) and in the nature of their change in a series of successive measurements, but significantly shortens the time interval of the above-described anomaly manifestation to the values observed in the experiments at low shear rates (Fig. 5).

A similar anomaly is also manifested in the flow curves in a wide $\left(0.5-200 \mathrm{~s}^{-1}\right)$ range of shear rates (Fig. 6). It occurs over a much longer time interval and can be easily seen when taking into account that the interval between adjacent points in the presented dependencies is 2 seconds. Already, during the second repetition of the experiment, the flow curves become unified (Fig. 6, curves 2, 3), which become typical for shear thinning and can be formally described, for example, by the classical Herschel-Bulkley equation [45]. Usually, such an experiment is not reproduced more than 2-3 times [46]. However, as it follows from the form of curve 4 in Fig. 6, the anomaly can reappear with an increase in the time interval between experiments. 


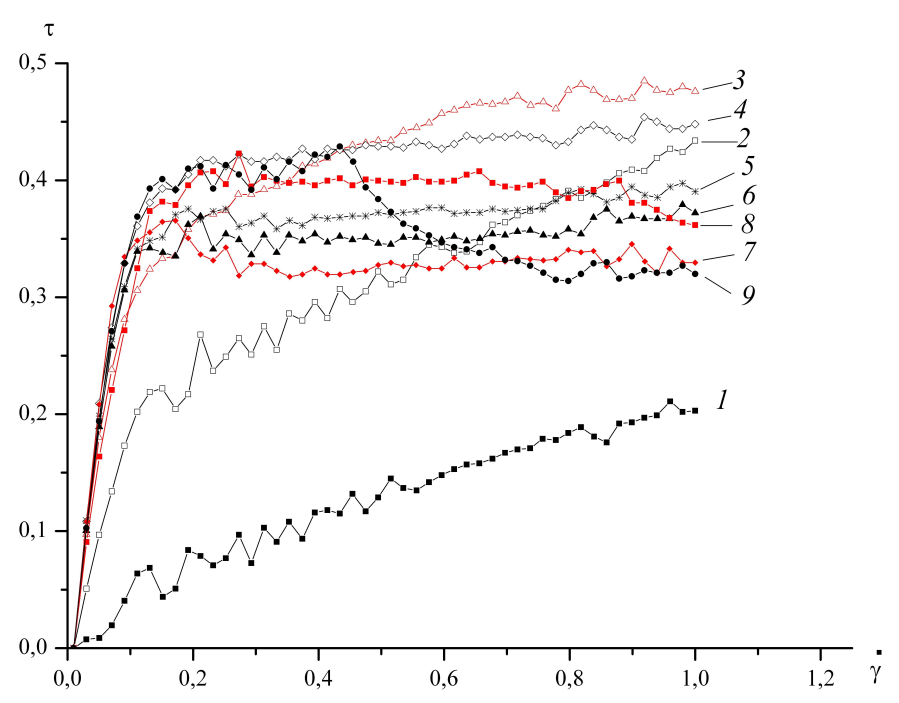

FIG. 8. $\tau(\mathrm{Pa})$ as a function of the shear rate $\gamma\left(\mathrm{s}^{-1}\right)$ for the sols obtained by precipitation in the region of low shear rates. Time intervals between adjacent measurements: 3 min

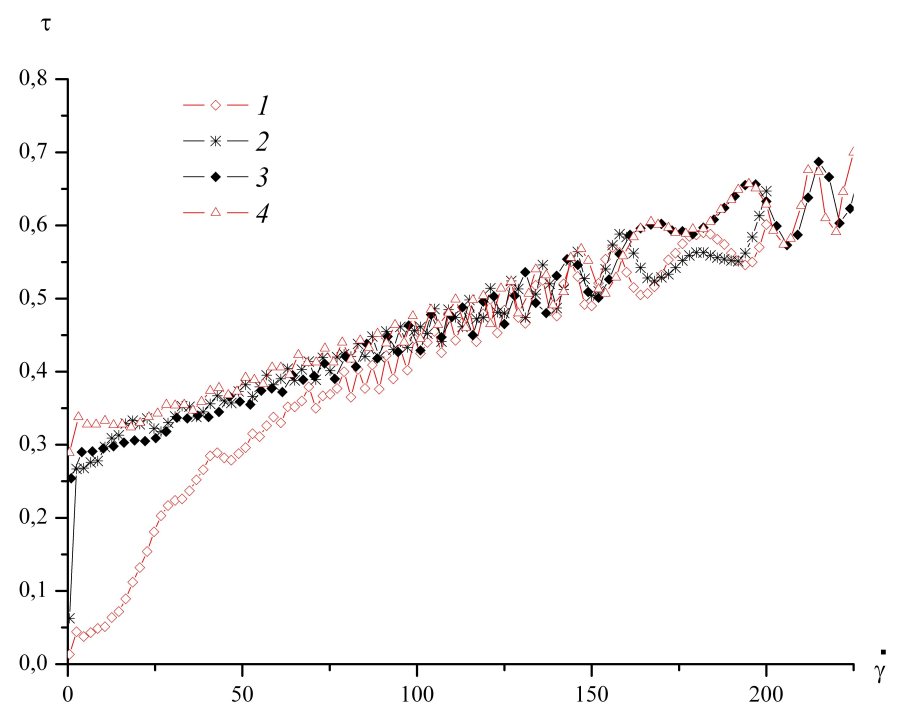

FIG. 9. $\tau(\mathrm{Pa})$ as a function of the shear rate $\gamma\left(\mathrm{s}^{-1}\right)$ for the sols obtained by precipitation in the broad range of shear rates. The first curve starts $7 \mathrm{~min}$ after the last measurement in the previous series (Fig. 8). Time intervals between adjacent measurements: 1-2 - 5 min; 2-3 - 4 min; 3-4 $13 \mathrm{~min}$

The behavior of the sols obtained by precipitation is completely different when mixing reagents in a glass and stirring on a magnetic stirrer. The corresponding flow curves are shown in Fig. 8. In the region of low shear rates (below $1 \mathrm{~s}^{-1}$ ), the first three flow curves are typical for shear thinning (Fig. 8, curves 1-3). Then the constant shear stress plateau appears (Fig. 8, curves 4-6), followed by the maximum shear stress (Fig. 8, curves 7-9). Therefore, it can be concluded that the nonequilibrium character of the flow intensifies along with the experiment reproduction.

Another difference from the behavior of sols obtained by the microreactor synthesis is in the absence of the flow curve anomalies over a broad range of shear rates (Fig. 9). Their appearance becomes unified and characteristic of the plastic flow already starting from the second repetition. And even a relatively long pause before the last measurement (Fig. 9, curve 4) does not cause a pronounced anomaly to appear, as was observed for the samples obtained through the microreactor synthesis. 


\section{Conclusion}

The presented data allow one to conclude that all the obtained lanthanum phosphate sols are structured systems, which are characterized by deformation behavior accompanied by shear thinning. The revealed anomalies associated with flow nonequilibrium at low shear rates indirectly indicate a stronger binding of particles in the structure of the samples obtained through the microreactor synthesis.

\section{Acknowledgements}

Rheological and X-ray diffraction studies, scanning electron microscopy and elemental analysis of samples were performed employing the equipment of the Engineering Center of the Saint Petersburg State Institute of Technology (Technical University). We thank our colleague D. P. Danilovich for his contribution to the project.

Structural characterization was made on the equipment of the Federal Joint Research Centre "Material science and characterization in advanced technology" (Ioffe Institute, Saint Petersburg, Russia). The authors would like to thank V. N. Nevedomsky for his assistance.

This work was supported by the Russian Foundation for Basic Research (project No 18-29-12119).

\section{References}

[1] Buissette V., Moreau M., Gacoin T., Boilot J.-P., Chane-Ching J.-Y., Le Mercier T. Colloidal Synthesis of Luminescent Rhabdophane $\mathrm{LaPO}_{4}: \mathrm{Ln}^{3+} \cdot x \mathrm{H}_{2} \mathrm{O}(\mathrm{Ln}=\mathrm{Ce}, \mathrm{Tb}, \mathrm{Eu} ; \mathrm{x} \sim 0.7)$ Nanocrystals. Chem. Mater, 2004, 16, P. 3767-3773.

[2] Fang Y.-P., Xu A.-W., Song R.-Q., Zhang H.-X., You L.-P., Yu J.C., Liu H.-Q. Systematic Synthesis and Characterization of Single-Crystal Lanthanide Orthophosphate Nanowires. J. Am. Chem. Soc., 2003, 125(51), P. 16025-16034.

[3] Glorieux B., Matecki M., Fayon F., Coutures J.P., Palau S., Douy A., Peraudeau G. Study of lanthanum orthophosphates polymorphism, in view of actinide conditioning. Journal of Nuclear Materials, 2004, 326(2-3), P. 156-162.

[4] Neupane M.R., Garrett G.A., Rudin S., Andzelm J.W. Phase dependent structural and electronic properties of lanthanum orthophosphate $\left(\mathrm{LaPO}_{4}\right)$. J. Phys. Condens. Matter, 2016, 28(20), P. 205501.

[5] Gausse C., Szenknect S., Qin D.W., Mesbah A., Clavier N., Neumeier S., Bosbach D., Dacheux N. Determination of the Solubility of Rhabdophanes $\mathrm{LnPO}_{4} \cdot 0.667 \mathrm{H}_{2} \mathrm{O}$ ( $\mathrm{Ln}=\mathrm{La}$ to Dy). Eur. J. Inorg. Chem., 2016, 28, P. 4615-4630.

[6] Osipov A.V., Mezentseva L.P., Drozdova I.A., Kuchaeva S.K., Ugolkov V.L., Gusarov V.V. Preparation and thermal transformations of nanocrystals in the $\mathrm{LaPO}_{4}-\mathrm{LuPO}_{4}-\mathrm{H}_{2} \mathrm{O}$ system. Glass Physics and Chemistry, 2009, 35(4), P. 431-435.

[7] Roncal-Herrero T., Rodríguez-Blanco J.D., Oelkers E.H., Benning L.G. The direct precipitation of rhabdophane $\left(\mathrm{REEPO}_{4} \cdot n \mathrm{H}_{2} \mathrm{O}\right)$ nano-rods from acidic aqueous solutions at 5-100 ${ }^{\circ}$ C. J. Nanopart. Res., 2011, 13, P. 4049-4062.

[8] Boakye E.E., Hay R.S., Mogilevsky P. Spherical Rhabdophane Sols. II: Fiber Coating. J. Am. Ceram. Soc., 2007, 90(5), P. 1580-1588.

[9] Mogilevsky P., Hay R.S., Boakye E.E., Keller K.A. Evolution of Texture in Rhabdophane-Derived Monazite Coatings. J. Am. Ceram. Soc., 2003, 86(10), P. 1767-1772.

[10] Kenges K.M., Proskurina O.V., Danilovich D.P., Aldabergenov M.K., Gusarov V.V. Synthesis and Properties of Nanocrystalline Materials Based on $\mathrm{LaPO}_{4}$. Russian Journal of Applied Chemistry, 2017, 90(7), P. 1047-1054.

[11] Sujith S.S., Arun Kumar S.L., Mangalaraja R.V., Peer Mohamed A., Ananthakumar S. Porous to dense LaPO 4 sintered ceramics for advanced refractories. Ceramics International, 2014, 40, P. 15121-15129.

[12] Arinicheva Y., Clavier N., Neumeier S., Podor R., Bukaemskiy A., Klinkenberg M., Roth G., Dacheux N., Bosbach D. Effect of powder morphology on sintering kinetics, microstructure and mechanical properties of monazite ceramics. Journal of the European Ceramic Society, 2018, 38(1), P. 227-234.

[13] Colomer M.T., Díaz-Guillén J.A., Fuentes A. Nanometric Sr-Doped $\mathrm{LaPO}_{4}$ Monazite: Synthesis by Mechanical Milling, Characterization, and Water Incorporation on its Structure. Journal of the American Ceramic Society, 2010, 93(2), P. 393-398.

[14] Sankar S., Raj A.N., Jyothi C.K., Warrier K.G.K., Padmanabhan P.V.A. Room temperature synthesis of high temperature stable lanthanum phosphate-yttria nano composite. Materials Research Bulletin, 2012, 47, P. 1835-1837.

[15] Shijina K., Sankar S., Midhun M., Firozkhan M., Nair B.N., Warrier K.G., Hareesh U.N.S. Very low thermal conductivity in lanthanum phosphate-zirconia ceramic nanocomposites processed using a precipitation-peptization synthetic approach. New J. Chem., 2016, 40, P. 5333-5337.

[16] Kim J., Cotte A., Deloncle R., Archambeau S., Biver C., Cano J.-P., Lahlil K., Boilot J.-P., Grelet E., Gacoin T. LaPO 4 Mineral Liquid Crystalline Suspensions with Outstanding Colloidal Stability for Electro-Optical Applications. Adv. Funct. Mater., 2012, 22(23), P. 4949-4956.

[17] Kim J., Martinelli L., Lahlil K., Boilot J.-P., Gacoin T., Peretti J. Optimized combination of intrinsic and form birefringence in oriented $\mathrm{LaPO}_{4}$ nanorod assemblies. Applied Physics Letters, 2014, 105(6), P. 061102.

[18] Kim J., Michelin S., Hilbers M., Martinelli L., Chaudan E., Amselem G., Fradet E., Boilot J.-P., Brouwer A.M., Baroud C.N., Peretti J., Gacoin T. Monitoring the orientation of rare-earth-doped nanorods for flow shear tomography. Nature Nanotechnology, 2017, 12(9), P. 914-919.

[19] Riwotzki K., Meyssamy H., Kornowski A., Haase M. Liquid-Phase Synthesis of Doped Nanoparticles: Colloids of Luminescing LaPO 4 :Eu and $\mathrm{CePO}_{4}: \mathrm{Tb}$ Particles with a Narrow Particle Size Distribution. J. Phys. Chem. B, 2000, 104(13), P. 2824-2828.

[20] Hay R.S., Boakye E.E., Mogilevsky P. Spherical Rhabdophane Sols. I: Rheology and Particle Morphology. J. Am. Ceram. Soc., 2007, 90(5), P. 1574-1579.

[21] Gavrichev K.S., Ryumin M.A., Tyurin A.V., Khoroshilov A.V., Mezentseva L.P., Osipov A.V., Ugolkov V.L., Gusarov V.V. Thermal behavior of $\mathrm{LaPO}_{4} \cdot n \mathrm{H}_{2} \mathrm{O}$ and $\mathrm{NdPO}_{4} \cdot n \mathrm{H}_{2} \mathrm{O}$ nanopowders. Journal of Thermal Analysis and Calorimetry, $2010,102, \mathrm{P} .809-811$.

[22] Maslennikova T.P., Osipov A.V., Mezentseva L.P., Drozdova I.A., Kuchaeva S.K., Ugolkov V.L., Gusarov V.V. Synthesis, mutual solubility, and thermal behavior of nanocrystals in the $\mathrm{LaPO}_{4}-\mathrm{YPO}_{4}-\mathrm{H}_{2} \mathrm{O}$ system. Glass Physics and Chemistry, 2010, 36(3), P. $351-357$. 
[23] Sankar S., Prajeesh G.P.V., Anupama V.N., Krishnakumar B., Hareesh P., Nair B.N., Warrier K.G., Hareesh U.N.S. Bifunctional lanthanum phosphate substrates as novel adsorbents and biocatalyst supports for perchlorate. Journal of Hazardous Materials, 2014,275 , P. $222-229$.

[24] Bryukhanova K.I., Nikiforova G.E., Gavrichev K.S. Synthesis and study of anhydrous lanthanide orthophosphate (Ln = La, Pr, Nd, Sm) nanowhiskers. Nanosystems: Phys. Chem. Math., 2016, 7(3), P. 451-458.

[25] Byrappa K., Murukanahally Kempaiah Devaraju, Paramesh J.R., Basavalingu B., Soga K. Hydrothermal synthesis and characterization of $\mathrm{LaPO}_{4}$ for bio-imaging phosphors. Journal of Materials Science, 2008, 43(7), P. 2229-2233.

[26] Colomer M.T., Zur L., Ferrari M., Ortiz A.L. Structural-microstructural characterization and optical properties of Eu ${ }^{3+}, \mathrm{Tb}^{3+}{ }_{-\mathrm{codoped}}$ $\mathrm{LaPO}_{4} \cdot n \mathrm{H}_{2} \mathrm{O}$ and $\mathrm{LaPO}_{4}$ nanorods hydrothermally synthesized with microwaves. Ceramics International, 2018, 44, P. 11993-12001.

[27] Colomer M.T., Delgado I., Ortiz A.L., Farinas J.C. Microwave-assisted Hydrothermal Synthesis of Single-crystal Nanorods of Rhabdophane-type Sr-doped $\mathrm{LaPO}_{4} \cdot n \mathrm{H}_{2}$ O. J. Am. Ceram. Soc., 2014, 97(3), P. 750-758.

[28] Runowski M., Grzyb T., Zep A., Krzyczkowska P., Gorecka E., Giersig M., Lis S. Eu ${ }^{3+}$ and Tb ${ }^{3+}$ doped LaPO 4 nanorods, modified with a luminescent organic compound, exhibiting tunable multicolour emission. RSC Adv., 2014, 4, P. 46305-46312.

[29] Stankiewicz A.I., Moulijn J.A. Process intensification: Transforming chemical engineering. Chem. Eng. Prog., 2000, 96(1), P. 22-34.

[30] Marchisio D.L., Rivautella L., Barresi A.A. Design and Scale-Up of Chemical Reactors for Nanoparticle Precipitation. AIChE Journal, 2006, 52(5), P. 1877-1887.

[31] Kumar R.D.V., Prasad B.L.V., Kulkarni A.A. Impinging Jet Micromixer for Flow Synthesis of Nanocrystalline MgO: Role of Mixing/Impingement Zone. Ind. Eng. Chem. Res., 2013, 52, P. 17376.

[32] Abiev R.Sh., Al'myasheva O.V., Gusarov V.V., Izotova S.G. Method of producing nanopowder of cobalt ferrite and microreactor to this end. RF Patent 2625981, Bull. N 20, 20.07.2017. https://patents.google.com/patent/RU2625981C1/en.

[33] Abiev R.S., Almyasheva O.V., Izotova S.G., Gusarov V.V. Synthesis of cobalt ferrite nanoparticles by means of confined impinging-jets reactors. J. Chem. Tech. App., 2017, 1(1), P. 7-13.

[34] Proskurina O.V., Nogovitsin I.V., Il'ina T.S., Danilovich D.P., Abiev R.Sh., Gusarov V.V. Formation of BiFeO3 Nanoparticles Using Impinging Jets Microreactor. Russian Journal of General Chemistry, 2018, 88(10), P. 2139-2143.

[35] Johnson B.K., Prud'homme R.K. Chemical Processing and Micromixing in Confined Impinging Jets. AIChE Journal, 2003, 49(9), P. 2264-2282.

[36] Ba-ldyga J., Jasińska M., Orciuch W. Barium Sulphate Agglomeration in a Pipe - An Experimental Study and CFD Modeling. Chemical Engineering \& Technology, 2003, 26(3), P. 334-340.

[37] Kenges K.M., Proskurina O.V., Danilovich D.P., Aldabergenov M.K., Gusarov V.V. Influence of the Conditions for Preparing LaPO 4 -Based Materials with Inclusions of the $\mathrm{LaP}_{3} \mathrm{O}_{9}$ Phase on Their Thermal and Mechanical Properties. Russian Journal of Applied Chemistry, 2018, 91(9), P. 1539-1548.

[38] Colomer M.T. Effect of $\mathrm{Sr}^{2+}$ doping on sintering behavior, microstructural development and electrical properties of LaPO${ }_{4} \cdot n \mathrm{H}_{2} \mathrm{O}$ nanorods prepared by dry mechanical milling. International Journal of Hydrogen Energy, 2018, 43(29), P. 13462-13474.

[39] Colomer M.T., Mosa J. Thermal Evolution, Second Phases, and Sintering Behavior of LaPO4nH2O Nanorods Prepared by Two Different Chemical Synthesis Routes. Ceramics International, 2015, 41(6), P. 8080-8092.

[40] Podval'naya N.V., Zakharova G.S., Liu Y. Phase Formation in the System $\mathrm{Zn}\left(\mathrm{VO}_{3}\right)_{2}-\mathrm{HCl}_{-} \mathrm{VOCl}_{2}-\mathrm{H}_{2} \mathrm{O}$. Russian Journal of Inorganic Chemistry, 2017, 62(8), P. 1104-1110.

[41] Krasilin A.A., Gusarov V.V. Redistribution of Mg and Ni cations in crystal lattice of conical nanotube with chrysotile structure. Nanosystems: Phys. Chem. Math., 2017, 8(5), P. 620-627.

[42] Zhou G. Study of hydrothermally synthesized $\mathrm{LiFePO}_{4}$ with different morphology. Russian Journal of Applied Chemistry, 2017, 90(9), P. 1519-1523.

[43] Mashentseva A.A., Kozlovskiy A.L., Turapbay K.O., Temir A.M., Seytbaev A.S., Zdorovets M.V. Determination of Optimal Conditions for Electoless Synthesis of Copper Nanotubes in the Polymer Matrix. Russian Journal of General Chemistry, 2018, 88(6), P. 1213-1218.

[44] Botman S.A., Leble S.B. Electrical conductivity model for quasi-one-dimensional structures. Nanosystems: Phys. Chem. Math., 2017, 8(2), P. 231-235.

[45] Herschel W.H., Bulkley R. Measurement of consistency as applied to rubber-benzene solutions. Am. Soc. Test. Proc., 1926, 26, P. 621-633.

[46] Schramm G. A Practical Approach to Rheology and Rheometry. 2-nd Ed. Gebrueder HAAKE GmbH, Karlsruhe, 2000,291 p. 